\title{
Micromagnetic Modeling of Head Field Rise Time for High Data-Rate Recording
}

\author{
Werner Scholz, Member, IEEE, and Sharat Batra, Member, IEEE
}

\begin{abstract}
We have developed a finite-element micromagnetics model to investigate the dynamics of write heads for perpendicular recording at high density and high data-rates. The model includes the entire head geometry, with the large return pole and the soft underlayer. The response of the head to the coil current is determined by the current waveform shape and duration, Gilbert damping constant, and presence of soft underlayer and shields. Large damping leads to a large phase shift between the coil current and the head field while small damping causes strong gyromagnetic precession. We find that an intermediate value of the damping constant gives the fastest head field rise time. The intrinsic reversal time decreases from 540 to 250 ps by reducing coil-turns from two to one and shortening the yoke length. Thus, an intermediate value of the damping constant, short yoke length, and fast current rise time are needed for maximum data rate.
\end{abstract}

Index Terms-Data-rate, dynamics, micromagnetics, perpendicular recording, write head.

\section{INTRODUCTION}

A S AREAL density and data rate in magnetic recording increase, the typical dimensions and time scales for various components in magnetic recording must decrease. As a result, an ever more detailed understanding of the behavior of magnetic recording media, read and write heads and the underlying physical effects is required. Finite-element models have been widely employed to design magnetic recording heads and calculate the write fields [1] and dynamic response [2]-[4]. However, as the size of the write pole shrinks, it is not only important to consider its intrinsic domain structure in the saturated and remanent state but also the dynamic micromagnetic processes which occur upon reversal of the coil current or during relaxation to remanence [2], [5], [6].

\section{Finite-Element Model}

We have developed a finite-element (FE) micromagnetic model to study magnetization reversal processes in write heads for perpendicular recording at high data rates.

First, the geometry (including the head, coils, return pole, and soft underlayer) and finite-element mesh are designed using a commercial FE electromagnetics package [7]. Within this commercial package we calculate the current density distribution in the coils for a given applied voltage and scale it to the desired coil current. Then we solve the Biot-Savart law to calculate the magnetic field, which is generated by the coil current, while the relative permeability is set everywhere to $\mu_{\mathrm{r}}=1$. Finally, the

Manuscript received July 23, 2004.

The authors are with Seagate Research, Pittsburgh, PA 15222 USA (e-mail: werner.scholz@seagate.com).

Digital Object Identifier 10.1109/TMAG.2004.839071

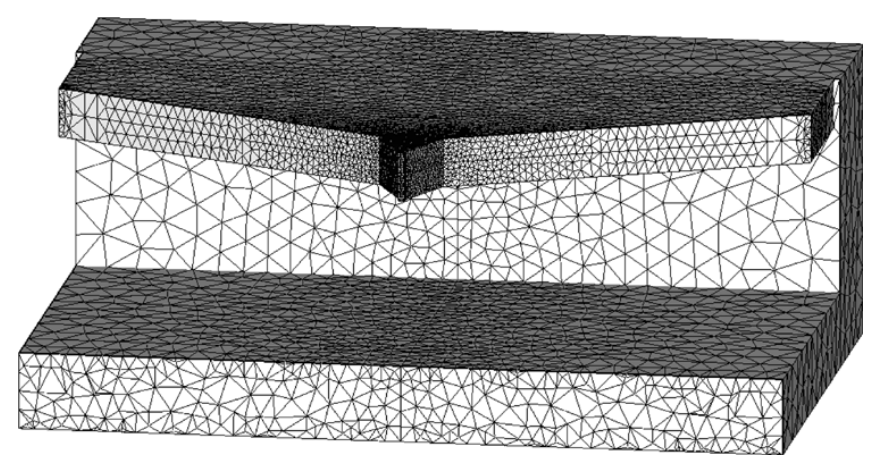

Fig. 1. Finite-element model of a SPT head for perpendicular recording.

FE mesh and the magnetic field of the coils are exported in a suitable format and imported into the FE micromagnetic model.

The micromagnetic model is based on a parallel finiteelement micromagnetics package [8], which is available under a free, open source license [9]. The model includes magnetocrystalline anisotropy, exchange interactions, external fields, and a hybrid finite-element/boundary-element method with a magnetic scalar potential for the calculation of the magnetostatic field. The energy minimization method is used to quickly obtain an initial domain configuration. Then, a preconditioned backward differentiation formula is used for the integration of the Landau-Lifshitz-Gilbert equation of motion for the magnetization to study dynamics. In order to model the coil (current), we import the field, which has been calculated by the electromagnetics program into the micromagnetic model and apply it as an external field. The linearity of the Biot-Savart law with respect to the current allows us to scale this field, so that any coil current and wave form can be simulated, albeit eddy current effects are neglected [2], [4]. Also, temperature effects have not been taken into account.

A model of a single pole write head for perpendicular recording is shown in Fig. 1. We have used a graded finite-element mesh with a resolution of about $15 \mathrm{~nm}$ around the pole tip and up to $300 \mathrm{~nm}$ in the pedestal and about 300000 tetrahedral finite elements with linear basis functions. We have also verified some results with a finer mesh with about one million finite elements and a mesh size down to $5 \mathrm{~nm}$. The model also includes a soft underlayer (SUL), which is not shown in Fig. 1. In the past it has been widely modeled as a magnetic mirror for simplicity [10], but the reversal of the SUL involves complex micromagnetic structures and the excitation of spin waves [11]-[13], which also affect the head dynamics. Therefore, we include a fully micromagnetic model of the SUL, which is also discretized into tetrahedral finite elements. However, the media layer has been omitted. 


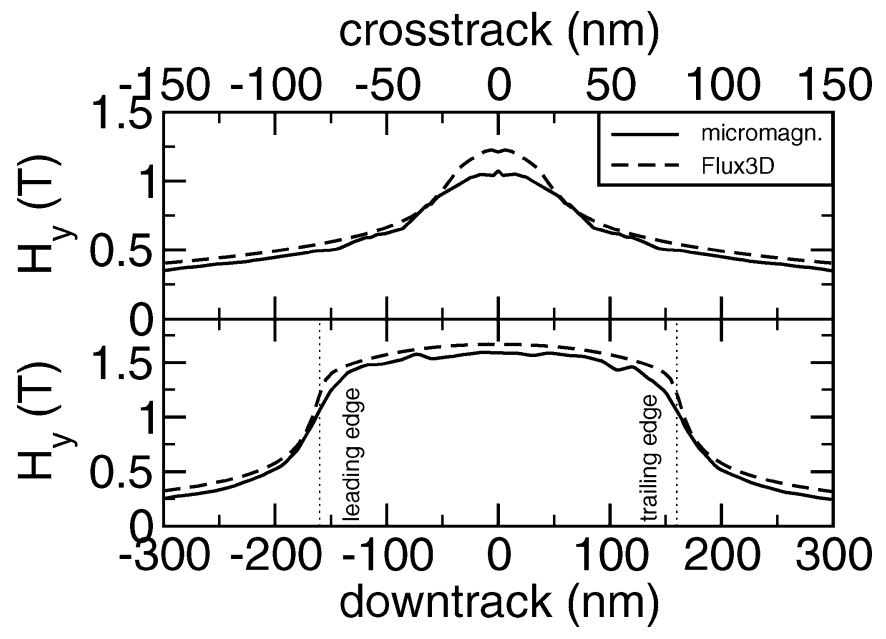

Fig. 2. Comparison of downtrack and crosstrack (measured at the trailing edge) field profiles between the micromagnetic and electromagnetic program (Flux3D) for an unshielded SPT head in the saturated state.

\section{StATIC FIELD PROFiles}

We have modeled a single pole type (SPT) head with a write pole width of $40 \mathrm{~nm}$, a pole thickness of $320 \mathrm{~nm}$, and a yoke length of $3.5 \mu \mathrm{m}$ [1]. The head and SUL parameters were chosen to maximize the head field, but they do not address other effects, such as skew. The head is energized by a single-turn coil, which is driven at a current of $150 \mathrm{~mA}$. The pole tip has a saturation magnetization of $M_{\mathrm{s}}=2.4 \mathrm{~T}$, an exchange stiffness of $13 \times 10^{-12} \mathrm{~J} / \mathrm{m}$, and an in-plane anisotropy of $K_{1}=$ $4800 \mathrm{~J} / \mathrm{m}^{3}$, which gives an anisotropy field of $H_{K}=50 \mathrm{Oe}$. The yoke and return pole have a saturation magnetization of $M_{\mathrm{s}}=1 \mathrm{~T}$, an exchange stiffness of $13 \times 10^{-12} \mathrm{~J} / \mathrm{m}$, and an in-plane anisotropy of $K_{1}=800 \mathrm{~J} / \mathrm{m}^{3}$, while the soft underlayer has a saturation magnetization of $M_{\mathrm{s}}=2 \mathrm{~T}$, an exchange stiffness of $10^{-11} \mathrm{~J} / \mathrm{m}$, and an in-plane anisotropy of $K_{1}=2400 \mathrm{~J} / \mathrm{m}^{3}$.

The remanent state is dominated by a vortex structure. For terabit-per-square-inch magnetic recording systems a head-tomedia spacing (HMS) of $5 \mathrm{~nm}$, a media thickness of $10-15 \mathrm{~nm}$, and a head-to-soft underlayer (HKS) spacing of 20-25 nm are anticipated [1], [14]. Thus, we chose HKS $=25 \mathrm{~nm}$, set the thickness of the SUL to $300 \mathrm{~nm}$ and measured the field in a plane at a distance of about $10 \mathrm{~nm}$ from the air-bearing surface (ABS). Fig. 2 shows a comparison of down-track and cross-track field profiles between the commercial FE electromagnetics program (where nonlinear material parameters with a saturation magnetization of $B_{\mathrm{s}}=2.4,1$, and $2 \mathrm{~T}$ have been assumed for the pole tip, the yoke and the soft underlayer, respectively) and the micromagnetic program. The electromagnetics program obtains a slightly higher downtrack field gradient at the trailing edge, which also leads to the difference in the maximum perpendicular field in the cross-track profile. Saturation of the head field has been found to occur at a current of about $100 \mathrm{~mA}$ through the single-turn coil.

\section{HEAD FIELD DYNAMICS}

After obtaining an initial magnetization distribution in zero field by static energy minimization, we excite the head with

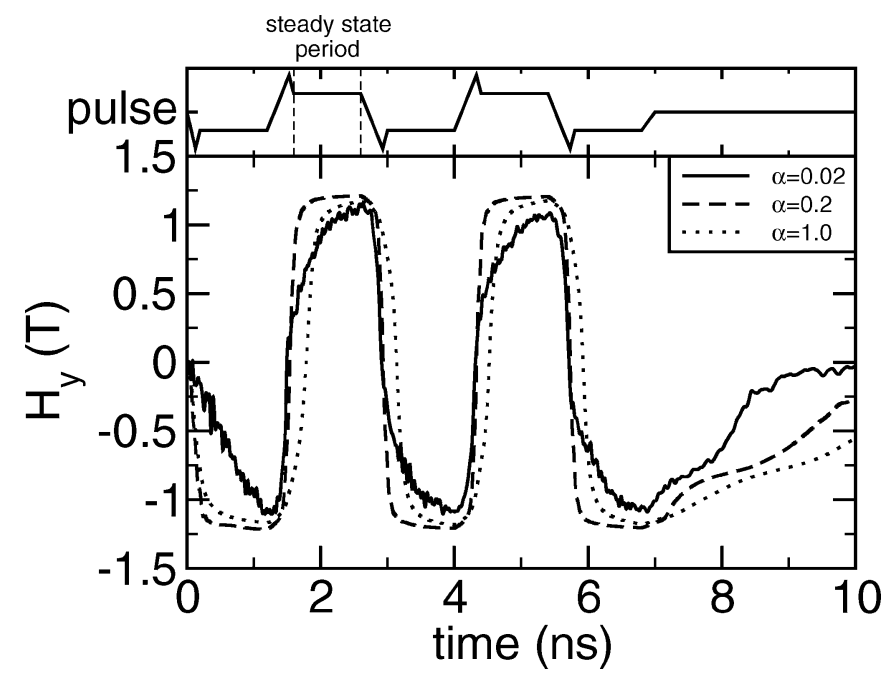

Fig. 3. Perpendicular field as a function of time for five current pulses with overshoot and different damping constants.

the magnetic field generated by the coils and integrate the Landau-Lifshitz-Gilbert equation of motion to obtain the time evolution of the magnetization. A single pulse of the coil current has a rise time of $0.13 \mathrm{~ns}$ from zero to the peak of the overshoot, and decays in 0.07 ns to its constant value, which is kept for 1 ns. Fig. 3 shows the field measured at the trailing edge, $8.3 \mathrm{~nm}$ from the ABS as a function of time for different damping constants. The head dynamics of the first pulse is not representative for the behavior of the head, because the initial magnetization distribution (domain structure) is still somewhat arbitrary. The damping constant is set to the same value everywhere in the model (including the SUL). For very small damping ( $\alpha=0.02$ ) the head demagnetizes quickly, but excessive gyromagnetic precession of the magnetization in the pole tip leads to a very slow approach to saturation [2], [15], [16]. As the Gilbert damping constant increases, the perpendicular field component reaches its maximum value much faster. However, in contrast to previous micromagnetic studies of longitudinal recording heads [2], [5] it also shows a considerable phase shift with respect to the current waveform, which has also been observed experimentally in longitudinal recording heads [17]. This phase shift leads to a shift of the recorded transitions [18].

The phase shift is shown in Fig. 4 for coil currents without overshoots as a function of damping constant. It is measured as the delay between the start of the current reversal (when the coil current starts to decrease), and the time when the head field drops by $15 \%$ (i.e., to $0.85 * 1.2 \mathrm{~T} \approx 1 \mathrm{~T}$ ). The phase shift increases monotonically with increasing damping constant and for $\alpha=1$ it reaches $0.53 \mathrm{~ns}$. This slow response becomes a problem for recording at high data rates, when the cell time for writing a single bit is of the same magnitude as the phase delay. Then, the head does not have enough time to generate maximum field to write the transition before the coil current is reversed, as will be shown next.

To study the influence of the steady-state period on head performance, we have applied current pulses with constant rise and decay times ( $0.2 \mathrm{~ns}$; total $0.4 \mathrm{~ns}$ peak to peak), but we have 


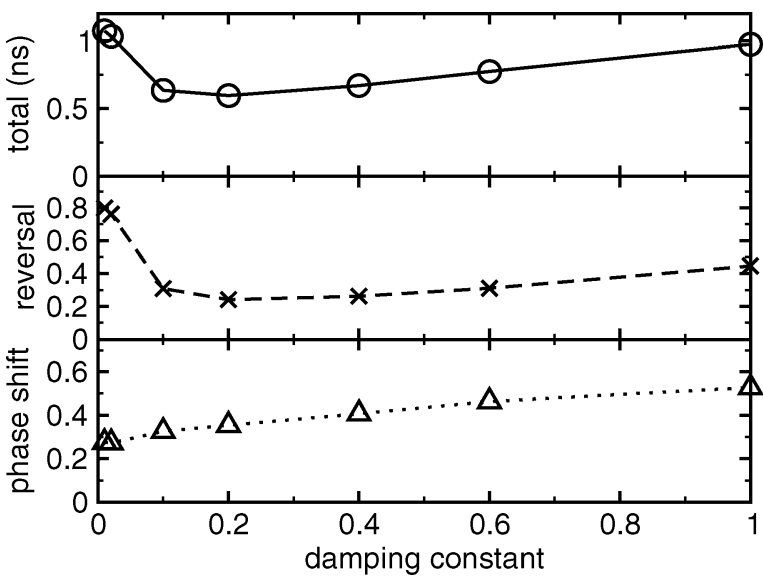

Fig. 4. Phase shift (delay between start of current reversal and $H_{y}=1 \mathrm{~T}$, i.e., drop by $15 \%$ from maximum field), reversal time (time between $H_{y}=1 \mathrm{~T}$ and $H_{y}=-1 \mathrm{~T}$, i.e., change from $+85 \%$ to $-85 \%$ of maximum field), total switching time (sum of phase shift and reversal time) as a function of damping constant for current pulses with $0.2 \mathrm{~ns}$ rise and $0.2 \mathrm{~ns}$ decay times $(0.4 \mathrm{~ns}$ peak to peak) without overshoot.

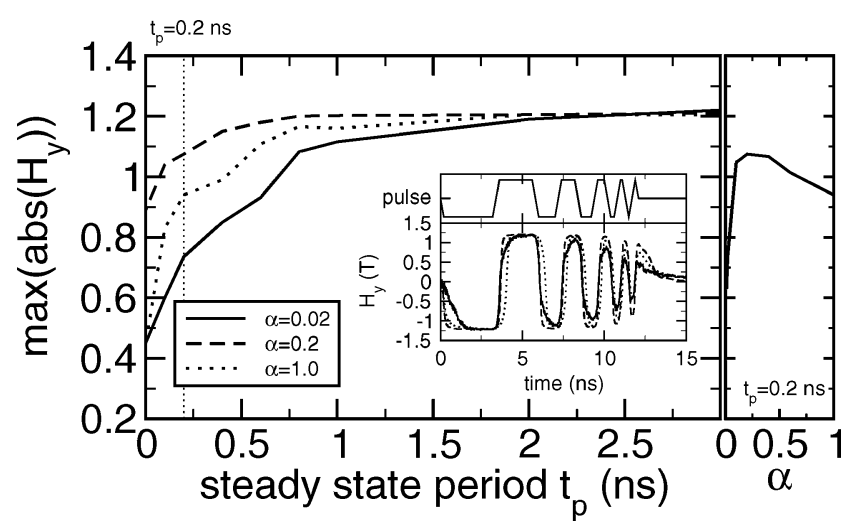

Fig. 5. Maximum perpendicular field as a function of steady-state period for different damping constants. The head was driven with a trapezoidal wave form with 0.2 ns rise time and a 0.2 ns decay time. The inset shows the current wave form and head field as a function of time for current pulses with decreasing cell time. The sidebar shows the maximum perpendicular field as a function of damping constant for a steady-state period $t_{\mathrm{p}}=0.2 \mathrm{~ns}$.

varied the steady-state period during which the current is kept constant. The consecutive pulses have a steady-state period of $3,2,1,0.8,0.6,0.4,0.2,0.1$, and $0 \mathrm{~ns}$, but no overshoot, corresponding to a cell time of 3.4 down to $0.4 \mathrm{~ns}$. The inset in Fig. 5 shows the perpendicular field for different damping constants. The maximum perpendicular field decreases for shorter cell times. Fig. 5 shows the maximum perpendicular field as a function of steady-state period for different damping constants. For large cell times the magnetization in the head has plenty of time to relax to a saturated state. Thus, the maximum field is independent of the damping constant and the head always produces its highest field. For $\alpha=0.01$ the maximum field for a steady-state period of $3 \mathrm{~ns}$ is even a little higher, because the ringing of the magnetization eventually produces slightly higher fields than in the final relaxed state, but these events occur for only very short periods of time $(<100 \mathrm{ps})$. For a steady-state period of $1 \mathrm{~ns}$ there is already a considerable spread in the maximum field. The sidebar in Fig. 5 plots the maximum field as a function of damping constant for a steady-state period of $0.2 \mathrm{~ns}$.
Heads with a damping constant of $\alpha=0.2$ (with our given geometry) produce the highest fields especially for small cell times and therefore high data rates.

In contrast, the low damping case shows a smaller phase shift, which indicates that the head demagnetizes quickly. However, it takes a very long time to approach saturation because of ringing of the magnetization. This fact is visible in Figs. 3 and 4. The intrinsic reversal time has been measured using a $15 \%-85 \%$ criterion: It is the time it takes the head to switch between a $15 \%$ reduced field (e.g., $1 \mathrm{~T}$ ) and $85 \%$ of the maximum field at the trailing edge in opposite direction (e.g., $-1 \mathrm{~T}$ ). Finally, the total switching time of the head is the sum of the phase shift and the intrinsic reversal time. Fig. 4 shows that the smallest switching time is obtained for $\alpha=0.2$, because heads with larger damping suffer from a larger phase shift, whereas heads with smaller damping require considerably more time to reach saturation.

From Fig. 5 we can deduce the data-rate limits for our head design for a given current pulse shape $(0.2$ ns rise time, no overshoot) for a given value of the damping constant and the media coercivity $\left(H_{K}\right)$ value. If the field requirement to write on the recording media is such that a perpendicular field $H_{y}$ of $1.2 \mathrm{~T}$ is needed, the shortest steady-state period must be at least $1 \mathrm{~ns}$ for all damping constant values. With rise and decay times of $0.2 \mathrm{~ns}$, the total steady-state period of $1.4 \mathrm{~ns}$ corresponds to a data-rate of $0.71 \mathrm{~Gb} / \mathrm{s}$. However, if a field of $H_{y}=1.1 \mathrm{~T}$ is sufficient to write the media, the steady-state period can be reduced to $0.6 \mathrm{~ns}$, for damping constant values greater than 0.02 . This results in a data rate of $1 \mathrm{~Gb} / \mathrm{s}$. High data-rate recording at $1 \mathrm{~Gb} / \mathrm{s}$ has been successfully demonstrated [19]. Media coercivity has to be decreased still further to write it at data-rates beyond $1 \mathrm{~Gb} / \mathrm{s}$. For a medium with good writability with a field of $H_{y}=1 \mathrm{~T}$, the steady-state period can be reduced to $0.1 \mathrm{~ns}$ (and thus the data-rate increased to $2 \mathrm{~Gb} / \mathrm{s}$ ), provided that the Gilbert damping constant is about 0.2. As a result, the tradeoff between high areal density and data rate will get worse for data rates beyond $1 \mathrm{~Gb} / \mathrm{s}$. At high data rate the head field is significantly reduced. This makes it harder to write on high coercivity media, which are needed to preserve thermal stability at high areal density. Since $\alpha=0.2$ gives the shortest switching time, it allows for the highest data-rates. Recent work on NiFe thin films shows the possibility of tailoring the damping constant by doping with rare-earth elements [20].

For a rectangular field pulse without overshoots (cf. Fig. 6) a shorter switching time is measured. However, this is not due to a faster intrinsic reversal of the head, but due to instantaneous turn-off of the current. During the time interval between the steady-state and zero current, the magnetization and the head field remain almost unaffected (indicating a slow decay to remanence as shown below). Only when the current switches its polarity and generates a field opposite to the magnetization of the head, the head starts to respond and the head field decays. The evolution of the field as a function of time is very similar for a $0.2 \mathrm{~ns}$ ramp or a rectangular pulse. The decay time of the previous pulse, which is $0.2 \mathrm{~ns}$, reduces to 0 for a rectangular pulse. Therefore, the field response is just shifted by $0.2 \mathrm{~ns}$ as shown in Fig. 6. The finite spin wave propagation speed in thin films [21] may be one of the contributing mechanisms to the phase delay due to the separation between the exciting coil and the 


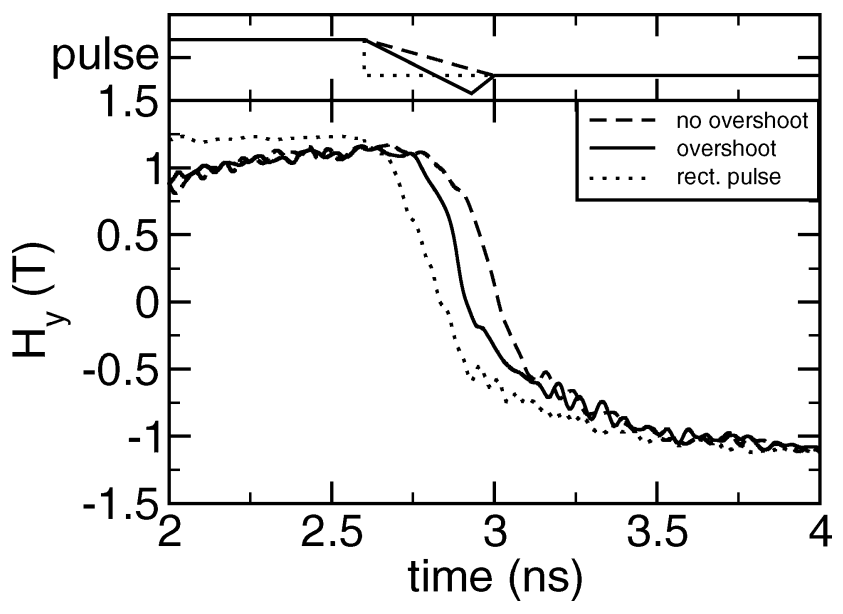

Fig. 6. Perpendicular field as a function of time for different current wave forms: dashed line: without current overshoot, solid line: with current overshoot, dotted line: rectangular current pulse.

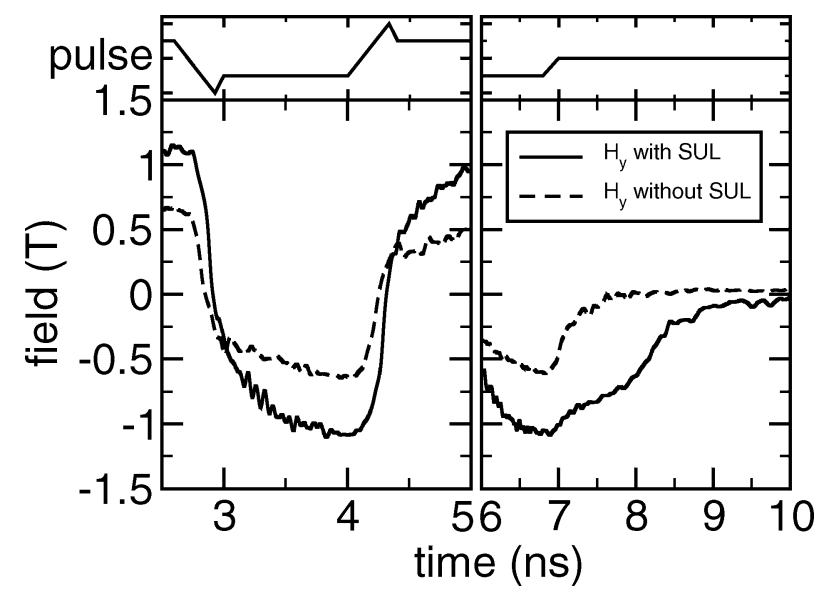

Fig. 7. Field dynamics with and without soft underlayer. The coil is switched off at $7 \mathrm{~ns}$.

pole tip. Thus, the current pulsewidth can be reduced to $0.3 \mathrm{~ns}$ cell time ( $0.2 \mathrm{~ns}$ phase shift plus $0.1 \mathrm{~ns}$ steady-state period), which increases the data rate from 2 to $3.3 \mathrm{~Gb} / \mathrm{s}$ in the example given above. This data-rate is based on the highest frequency that the head can be switched between two states, and the head generates a perpendicular field $H_{y}= \pm 1 \mathrm{~T}$. This performance is consistent with the predictions of previous studies for the response time of magnetic recording heads [22], [23], though, the media would have to support these data-rates with fast switching speeds, too [24], [25].

The effect of current overshoot on a head with $\alpha=0.02$ is also shown in Fig. 6. The current wave form with overshoot switches the polarity of the current faster, which reduces the phase shift by $0.06 \mathrm{~ns}$ for a system with $\alpha=0.02$. Thus, fast switching of the coil current allows for higher data rates.

Another important aspect is the interaction of the head with the SUL. In the energized state of the head, the SUL collects the magnetic flux from the head, and channels it to the return pole, effectively becoming part of the magnetic circuit. Its effect as a magnetic mirror, enhances the magnetic field of the write pole, but its dynamics and interaction with the head cannot be neglected. Fig. 7 shows the head field as a function of time for

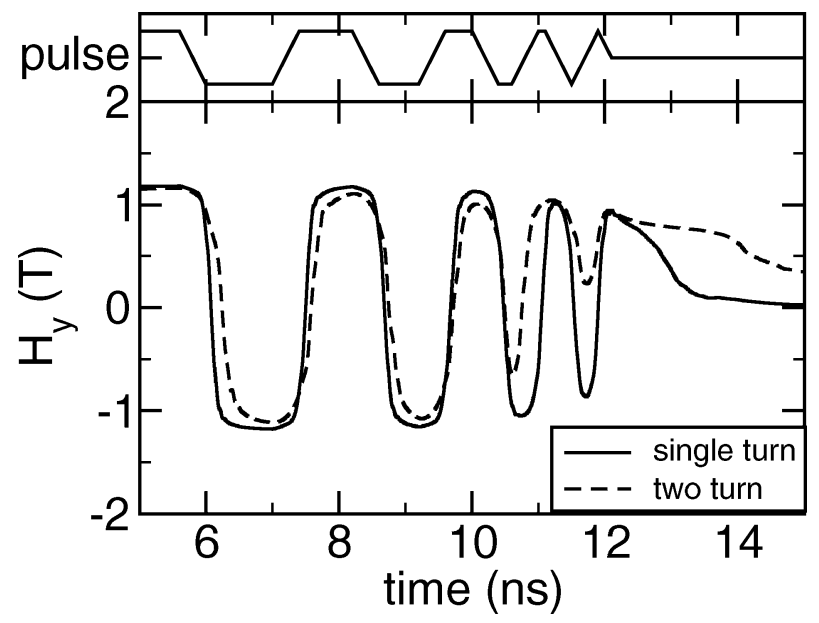

Fig. 8. Head field response for a write head with a single-turn (black lines) and a two-turn coil (dashed lines). The damping constant of the system is $\alpha=0.2$ in both cases.

a system with and without SUL. The SUL increases the maximum field from 0.65 to $1.1 \mathrm{~T}$, but its interaction with the head also slows down the dynamics of the head. The model with SUL shows a larger phase shift than the system without SUL. Moreover, the decay to remanence (when the coils are switched off at $t=7 \mathrm{~ns}$ ) is slowed down by the SUL. It takes almost $2 \mathrm{~ns}$ until the perpendicular field $H_{y}$ drops below $0.1 \mathrm{~T}$ (cf. Fig. 7). Detached pole tip designs and laminated head structures have been proposed to speed up the head response [6], [10].

Finally, we checked the influence of the number of turns and yoke length on the switching time of the head. To accommodate the second turn, we have increased the yoke length to $7 \mu \mathrm{m}$ and energized the coils with a current of $75 \mathrm{~mA}$ each, thus preserving the magnetomotive force of $150-\mathrm{mA}$ turns. Fig. 8 shows the perpendicular head field as a function of time for a single-turn and a two-turn head with $\alpha=0.2$. The phase shift is affected little, because the distance of the front turn of the coil from the ABS remained the same. However, the intrinsic reversal time increases from 250 to $540 \mathrm{ps}$, because the front turn is carrying only half the current (as compared to the single-turn case) and the second turn is shifted by $3.5 \mu \mathrm{m}$ toward the back. Thus, for high data-rate recording, head designs with short yokes and single-turn coils with high currents close to the pole tip are advantageous [26], [27].

\section{CONCLUSION}

We have studied the reversal dynamics of perpendicular write heads for high density and high data-rates. It has been found, that the response time and field of the head strongly depend on the damping constant of the system. The phase shift of the head field relative to the current waveform through the coils increases for increasing damping constant values. If the cell time is comparable to the phase-shift, the head cannot generate its maximum field before the current is reversed. For low damping, the phase shift is much smaller, but gyromagnetic precession of the magnetization in the pole tip leads to a slow approach to saturation. The shortest switching time and highest data-rate was found for a Gilbert damping constant of 0.2 for our head geometry. Head designs with short yoke length, single-turn coils 
with high currents close to the pole tip, and current drivers with fast current rise time and overshoot will be required to obtain the fastest field rise time for the head. In addition, the head field is significantly lower at short cell times. A lower head field will limit the achievable areal density on thermally stable media.

\section{ACKNOWLEDGMENT}

The authors wish to thank H. Zhou, T. Roscamp, and P. Asselin for helpful discussions and M. Crawford for review of the manuscript.

\section{REFERENCES}

[1] S. Batra, J. D. Hannay, H. Zhou, and J. S. Goldberg, "Investigations of perpendicular write head design for $1 \mathrm{~Tb} / \mathrm{in}^{2}$," IEEE Trans. Magn., vol. 40, no. 1, pp. 319-325, Jan. 2004.

[2] K. Takano, "Magnetization dynamics of planar writers," IEEE Trans. Magn., vol. 40, no. 1, pp. 257-262, Jan. 2004.

[3] Y. Kanai, R. Matsubara, H. Watanabe, H. Muraoka, and Y. Nakamura, "Finite-element model analysis of single-pole-type head for $1 \mathrm{Tbit} / \mathrm{in}^{2}$," IEEE Trans. Magn., vol. 39, no. 5, pp. 2405-2407, Sep. 2003.

[4] Y. Kanai and R. Matsubara, "Write field calculation for a narrow-track, single-pole head with a thin underlayer of perpendicular medium," IEEE Trans. Magn., vol. 38, no. 1, pp. 169-174, Jan. 2002.

[5] J.-G. Zhu, C. Y. Mao, R. M. White, S. Batra, and R. Rottmayer, "Understanding single-turn write head design at narrow track widths," IEEE Trans. Magn., vol. 38, no. 1, pp. 124-128, Jan. 2002.

[6] C. Y. Mao, J.-G. Zhu, R. M. White, and T. Min, "Effect of damping constant on the switching limit of a thin-film recording head," J. Appl. Phys., vol. 85, no. 8, pp. 5870-5872, Apr. 1999.

[7] Flux3D, Magsoft Corp., Troy, NY, pp. 12 180-12 180.

[8] W. Scholz, J. Fidler, T. Schrefl, D. Suess, R. Dittrich, H. Forster, and V. Tsiantos, "Scalable parallel micromagnetic solvers for magnetic nanostructures," Comp. Mat. Sci., vol. 28, pp. 366-383, 2003.

[9] Magpar - Parallel Finite Element Micromagnetics Package [Online]. Available: http://magnet.atp.tuwien.ac.at/scholz/magpar/

[10] D. Z. Bai and J.-G. Zhu, "A detached pole tip design of perpendicular write heads for high data-rate recording," IEEE Trans. Magn., vol. 38, no. 5, pp. 2240-2242, Sep. 2002.

[11] D. Litvinov et al., "Micromagnetics of a soft underlayer," IEEE Trans. Magn., vol. 36, no. 5, pp. 2483-2485, Sep. 2000.
[12] M. E. Schabes, B. Lengsfield, and T. Schrefl, "Micromagnetic modeling of soft underlayer magnetization processes and fields in perpendicular magnetic recording," IEEE Trans. Magn., vol. 38, no. 4, pp. 1670-1675, Jul. 2002.

[13] T. Schrefl, M. E. Schabes, and B. Lengsfield, "Fast reversal dynamics in perpendicular magnetic recording media with soft underlayer," J. Appl. Phys., vol. 91, no. 10, pp. 8662-8664, May 2002.

[14] M. Mallary, A. Torabi, and M. Benakli, "One terabit per square inch perpendicular recording conceptual design," IEEE Trans. Magn., vol. 38, no. 4, pp. 1719-1724, Jul. 2002.

[15] M. S. Patwari and R. H. Victora, "Unshielded perpendicular recording head for $1 \mathrm{~Tb} / \mathrm{in}^{2}$," IEEE Trans. Magn., vol. 40, no. 1, pp. 247-252, Jan. 2004.

[16] K. Z. Gao and H. N. Betram, "3-D micromagnetic simulation of write field rise time in perpendicular recording," IEEE Trans. Magn., vol. 38, no. 5, pp. 2063-2065, Sep. 2002.

[17] M. R. Freeman and J. F. Smyth, "Picosecond time-resolved magnetization dynamics of thin-film heads," J. Appl. Phys., vol. 79, no. 8, pp. 5898-5900, Apr. 1996.

[18] R. Wood and M. Williams, "Considerations for high data rate recording with thin-film heads," IEEE Trans. Magn., vol. 26, no. 6, pp. 2954-2959, Nov. 1990.

[19] F. H. Liu, K. Stoev, L. Leal, J. Wang, Y. Chen, S. Shi, H. C. Tong, M. Lederman, and M. Re, "Advanced heads for perpendicular recording at high areal densities and high data rates," IEEE Trans. Magn., vol. 38, no. 1, pp. 151-156, Jan. 2002.

[20] W. Bailey, P. Kabos, F. Mancoff, and S. Russek, "Control of magnetization dynamics in $\mathrm{Ni}_{81} \mathrm{Fe}_{19}$ thin films through the use of rare-earth dopants," IEEE Trans. Magn., vol. 37, no. 4, pp. 1749-1754, Jul. 2001.

[21] M. Covington, T. M. Crawford, and G. J. Parker, "Time-resolved measurement of propagating spin waves in ferromagnetic thin films," Phys. Rev. Lett., vol. 89, no. 23, p. 237 202, Dec. 2002.

[22] R. Wood, J. Miles, and T. Olson, "Recording technologies for terabit per square inch systems," IEEE Trans. Magn., vol. 38, no. 4, pp. 1711-1718, Jul. 2002.

[23] R. Wood, "The feasibility of magnetic recording at 1 Terabit per square inch," IEEE Trans. Magn., vol. 36, no. 1, pp. 36-42, Jan. 2000.

[24] M. E. Schabes, H. Zhou, and H. N. Bertram, "Effects of head field pulse shape and rise time on subnanosecond magnetization reversal in longitudinal thin film media," J. Appl. Phys., vol. 87, pp. 5666-5668, May 2000.

[25] M. Benakli, A. F. Torabi, M. L. Mallary, H. Zhou, and H. N. Bertram, "Micromagnetic study of switching speed in perpendicular recording media," IEEE Trans. Magn., vol. 37, no. 4, pp. 1564-1566, Jul. 2001.

[26] J. Jury, A. Gopinath, and J. H. Judy, "Design of a single-turn microstrip write head for ultra-high data rate recording," IEEE Trans. Magn., vol. 35, no. 5, pp. 2547-2549, Sep. 1999.

[27] S. Batra et al., "A perpendicular write head design for high-density recording," IEEE Trans. Magn., vol. 38, no. 1, pp. 157-162, Jan. 2002. 